\title{
Üçüncü derece zaman gecikmeli sistemler için PI denetleyicilerin analitik tasarımı
}

\section{Analytical design of PI controllers for third order plus time delay systems}

\author{
Bilal ŞENOL $1^{*}$ iD \\ ${ }^{1}$ Bilgisayar Mühendisliği Bölümü, Mühendislik Fakültesi, İnönü Üniversitesi, Malatya, Türkiye. \\ bilal.senol@inonu.edu.tr
}

Geliş Tarihi/Received: 11.03.2019

Düzeltme Tarihi/Revision: 15.08.2019

doi: $10.5505 /$ pajes.2019.19616

Kabul Tarihi/Accepted: 11.09.2019 Araștırma Makalesi/Research Article

$\ddot{0} \mathbf{z}$

Bu yayında üçüncü derece ve zaman gecikmesi içeren sistem modellerinin kararlılığı, performansı ve dayanıklılığı için analitik bir oransal integral denetleyici tasarım yöntemi sunulmustur. Denetleyici tasarımı, ideal bir sistemin frekans tepkilerini ele alarak denetlenmek istenen sistemin arzu edilen kazanc ve faz özelliklerini sağlaması üzerinde yoğunlaşmış̧ır. Sözü edilen özellikleri sağlayan oransal integral denetleyici parametrelerini veren denklemler adım adım olusturulmustur. $\mathrm{Bu}$ denklemler, söz konusu sistemler için genelleștirilmiş eșitlikleri içermektedir. Araștırmacı, bu yöntem sayesinde üçüncü derece zaman gecikmeli sistemler için istenen kazan kesim frekansı ve faz payı değerlerini sağlayabilmektedir. Bu sayede, Bode grafiği nispeten ayarlanabilmekte ve sistemin performansı ve dayanıklılığı artırılabilmektedir. Aynı zamanda önerilen denklemlerle sistem kararlığı da elde edilebilmektedir. Yayında sunulan yöntemle elde edilen denklemler iki farklı model üzerinde uygulanmıștır. Tüm sonuçlar grafiksel olarak ve tablolarla gösterilmiștir.

Anahtar kelimeler: PI denetleyici, TOPTD model, Kazanç ve faz özellikleri.

\section{Giriş}

Oransal Integral Türev (Proportional Integral Derivative, PID) denetleyiciler, olumlu etkileri, kurulum ve kullanım kolaylıkları ile endüstride yaygın olarak kullanılmaktadır. Bu yapının etkin kullanımı, aynı zamanda Oransal Integral (Proportional Integral, PI) denetleyicilerin ortaya çıkmasına sebep olmuştur. Son yıllarda PI denetleyicilerin tasarımını konu alan çalıșmaların sayısında belirgin bir artış olmuştur.

Zaman gecikmesi içeren modellerle ilgili çalıșmalara literatürde sıkça rastlanmaktadır. Birinci derece zaman gecikmeli (First Order plus Time Delay, FOPTD) ve İkinci derece zaman gecikmeli (Second Order plus Time Delay, SOPTD) modeller için çok sayıda denetleyici tasarım yöntemi sunulmuștur. Fakat üçüncü derece zaman gecikmeli (Third Order plus Time Delay, TOPTD) modeller ile ilgilenen çalışmalara daha az rastlanmaktadır. Doğrudan söz konusu sistemlerle ilgili çalışmalara pek rastlanmasa da, TOPTD ile ifade edilen bazı gerçek süreçler bulmak mümkündür. Örnek olarak [1]'de, elektriksel iletkenlik modeli TOPTD bir transfer fonksiyonu ile ifade edilmiştir. [2]'de, bir hava ısıtıcının sistem cevabı üçüncü derece bir modele uydurulmuştur. TOPTD model ile ifade edilen Hammerstein-Wiener süreci [3]'te verilmiştir. Küspe ile ateşlenen bir kaynatıcı kazanındaki buhar basıncının modellenmesi de [4] örnek olarak verilebilir. Söz konusu
Abstract

This paper presents an analytical method to design proportional integral controllers for the stability, performance and robustness of third order plus time delay system models. Controller design is focused on providing desired gain and phase specifications of the controlled system by considering the frequency responses of an ideal system. Equations that give the parameters of the proportional integral controller which satisfy the mentioned properties are built step by step. These include the generalized equations for such systems. With the help of this method, the researcher can provide desired gain crossover frequency and the phase margin for third order plus time delay systems. Therefore, the Bode diagram can be relatively tuned and system performance and robustness can be improved. At the same time stability of the system can be achieved with the proposed equations. Proposed controller parameters in this paper are applied on two different models. All results are shown graphically and on tables.

Keywords: PI Controller, TOPTD model, Gain and phase specifications.

modeller için denetleyici tasarımı da oldukça ilgi gören konular arasındadır. Bu konudaki bazı çalışmalardan yüksek dereceli salınımlı sistemler için geliştirilmiş performanslı PID denetleyici tasarımı için bir çalışma [5]'te verilmiştir. İki farklı darbe sinyali girișli FOPTD sistemlerin tanımlanması [6]'da bulunabilir. Bu çalışma TOPTD modeller için de uygulanmıştır. Kritik sönümlü SOPTD sistemler için geliştirilmiş PID denetleyici tasarım [7], basamak ve darbe tepki yöntemi ile Wiener süreçlerinin tanımlanması [8], frekans cevaplarının tahmini için geliștirilmiș Fourier dönüșüm yöntemi [9], zaman gecikmeli sistemler için doğrudan yöntemle frekans bölgesinde PID denetleyici tasarımı [10] TOPTD sistemler için uygulanan çalışmalar olarak gösterilebilir. Ayrıca, yüksek dereceli doğrusal modellerin bilgisayar yardımıyla sadeleștirilmesi bu konudaki önemli çalışmalardan biridir [11].

Yukarıda da söz edildiği gibi PI denetleyiciler literatürde oldukça geniș kullanım alanı bulmaktadır. Aktif süspansiyon sistemi için kayan kipli PI denetleyici tasarımı [12]'de verilmiștir. DC motor hız kontrolü için PI ve integral oransal (Integral Proportional, IP) denetleyicilerin karşılaştırmalı tasarım yöntemi [13]'te çalıșılmıştır. Tek bölge yük frekanshı kontrol sistemi için PI denetleyicinin parametre uzayındaki kararlılık bölgesi ile ilgili bir çalıșma da [14]'te bulunabilir. Tip2 bulanık mantık ile zamanla değișen PI denetleyicilerin elektrikli araçların hız kontrolü üzerine uygulanması [15]'de

*Yazışılan yazar/Corresponding author 
verilmiştir. Sabit mıknatıslı senkron motorların hız kontrolü için uyarlamalı bulanık mantık denetleyici ve PI denetleyici karşılaştırması [16]'da, PI denetleyici kullanılarak sabit mıknatıslı senkron motorların tork dalgacık minimizasyonu [17]'de görülebilir. Bu liste daha başka değerli çalışmalar eklenerek uzatılabilir.

$\mathrm{Bu}$ çalışmada üçüncü derece zaman gecikmeli sistem modellerinin kararlılık, performans ve dayanıklılığı için PI denetleyici tasarımı yapılmıştır. Tasarımdaki amaç, frekans bölgesinde iki özellik olan kazanç kesim frekansı ve faz payını istenildiği gibi ayarlayabilmektir. Böylece sistem performansı ve dayanıklılığı da nispeten ayarlanmıș olacaktır. Daha önce benzer doğrultuda yapılmış çalıșmalar bulmak mümkündür. Örnek olarak [18]'deki, zaman gecikmeli kararlı ve kararsız sistemlerin frekans bölgesinde PID denetleyici tasarımı çalışması verilebilir. Sistem kararlılığı için kararlılık sınır eğrileri yöntemi (Stability Boundary Locus, SBL) ile PI ve PID denetleyici tasarımı [19]'da ve zaman ve frekans bölgesi özelliklerinin aynı anda sağlanması için PI denetleyici tasarımı [20]'de bulunabilir. Yine zaman gecikmeli modeller için kesir dereceli PI ve oransal türev (Proportional Derivative, PD) denetleyicilerin frekans özelliklerine göre tasarımı örnek olarak verilebilir [21],[22]. Benzer çalışmaların çoğu sistem dayanıklılığını artırmak için Bode grafiğinde kazanç kesim frekansından sonraki faz eğrisini düzleștirme üzerine yoğunlaşmıştır [23],[24]. Bu çalışmada ise faz payını istenilen şekilde ayarlayarak sistem dayanıklılığına katkıda bulunmak hedeflenmiştir. Yöntemin özgünlüğü, doğrudan kazanç kesim frekansı ve faz payı tanımlarından yola çıkılarak denklemlerin elde edilmesinden gelmektedir. Ayrıca önerilen denklemlerin analitik olarak elde edilmiş olması da avantajlardan birisidir.

Yayının geri kalanı şu şekilde düzenlenmiștir. 2. Bölümde TOPTD sistem modeli, PI denetleyici ve tasarımda baz alınan frekans özellikleri verilmiştir. Ayrıca bu bölümde hatırlatma amacıyla Bode grafiği üzerinde bazı bilgilere yer verilmiștir. 3 . Bölümde önceden belirlenen frekans özellikleri ele alınarak PI denetleyici tasarımı yapılmış ve 4. Bölümde iki adet örnek TOPTD model üzerinde önerilen denklemler uygulanmıştır. Son bölüm ise sonuçlar ve tartışma bölümüdür.

\section{TOPTD sistemler, PI denetleyiciler ve tasarım özellikleri}

$\mathrm{Bu}$ bölümde önce TOPTD sistemler ve PI denetleyiciler tanıtılmıştır. Daha sonra ise ideal bir sistemin sağlaması istenen özelliklere yer verilmiştir. Zaman gecikmeli bir TOPTD model aşağıdaki gibi verilebilir.

$$
P(s)=\frac{K}{\left(T_{1} s+1\right)\left(T_{2} s+1\right)\left(T_{3} s+1\right)} \mathrm{e}^{-L s}
$$

Burada, $K$ model kazancl, $T_{i}$ zaman sabitleri ve $L$ gecikme terimidir. Giriș bölümünde belirtildiği gibi bu model gerçek süreçlerin ifade edilmesinde de kullanılmaktadır [1]-[4]. Bu modelin denetlenmesi için tasarımı gerçekleştirilmek istenen PI denetleyicinin genel gösterimi ise aşağıdaki gibidir.

$$
C(s)=k_{p}+\frac{k_{i}}{s}=\frac{k_{p} s+k_{i}}{s}
$$

Yukarıda verilen üçüncü derece model ve denetleyiciden oluşan sistem aşağıda gösterilmiştir.

$$
G(s)=C(s) P(s)
$$

Sistemin kapalı çevrim gösterimi de Şekil 1'de verilmiștir.

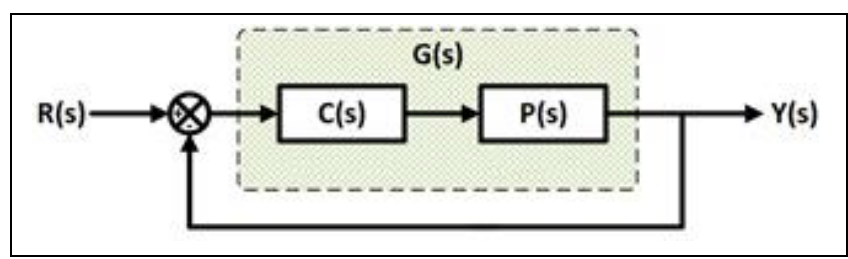

Şekil 1. Kapalı çevrim sistem.

Figure 1. Closed loop system.

Tasarım yöntemi frekans tabanlı bir yöntem olduğu için bazı bilgileri vermekte yarar vardır. Bir sistemin frekans bölgesinde analizini yapabilmek için sistem transfer fonksiyonunda $s$ yerine $j \omega$ yazılarak işlemlere devam edilmelidir. Denklem (1)'de verilen modelin ve PI denetleyicinin frekans bölgesi gösterimleri aşağıda verilmiştir.

$$
\begin{gathered}
P(j \omega)=\frac{K}{\left(T_{1}(j \omega)+1\right)\left(T_{2}(j \omega)+1\right)\left(T_{3}(j \omega)+1\right)} \mathrm{e}^{-L(j \omega)} \\
C(j \omega)=k_{p}+\frac{k_{i}}{j \omega}=k_{p}-\frac{j k_{i}}{\omega}
\end{gathered}
$$

Benzer şekilde, açık çevrim sistem frekans bölgesinde aşağıdaki şekilde elde edilebilir.

$$
G(j \omega)=C(j \omega) P(j \omega)
$$

Örnek bir Bode diyagramı Şekil 2'de gösterilmiştir.

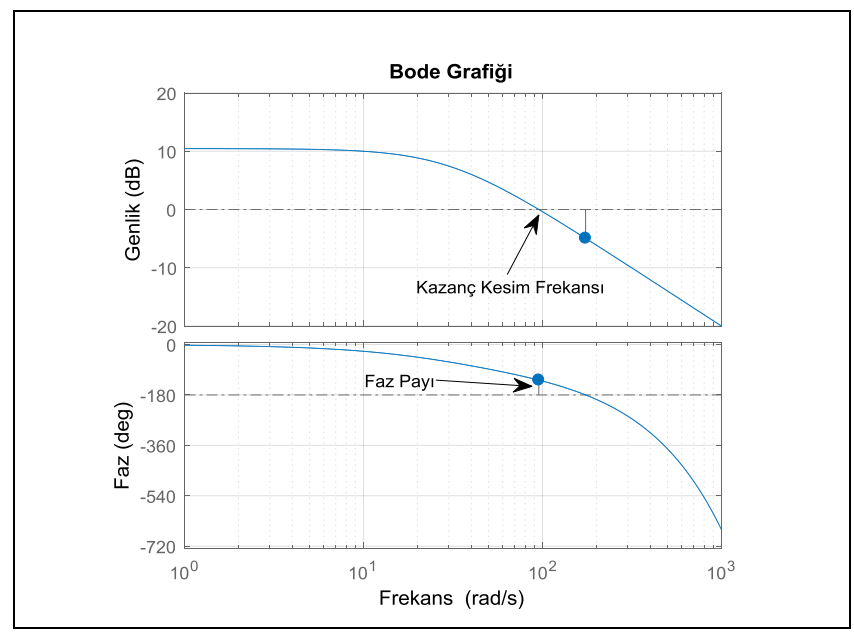

Şekil 2. Örnek bir Bode diyagramı.

Figure 2. An example Bode diagram.

Bode diyagramı desibel cinsinden genlik ve derece cinsinden faz eğrilerinden oluşmaktadır. Genlik eğrisinin 0 çizgisini kestiği frekans değeri kazanç kesim frekansı olarak adlandırılmaktadır ve bu yayında $\omega_{c}$ olarak kullanılmıştır. Kazanç kesim frekansında faz eğrisinin $-180^{\circ}$ çizgisine olan uzaklığı da faz payıdır ve bu yayında $\phi_{m}$ olarak verilmiştir. Sistemlerde faz payının değeri sistem performansı ve dayanıklılığına etki etmektedir. $\mathrm{Bu}$ da, bu konuda yapılan çalışmaların önemini ortaya koymaktadır. 
$\mathrm{Bu}$ yayında kullanılan frekans özellikleri ideal bir sistemin frekans cevabından esinlenerek ele alınmıştır. Așağıda verilen kazanç ve faz özelliklerinin sağlanması amaçlanmaktadır.

$$
\begin{gathered}
\left|G\left(j \omega_{c}\right)\right|=1 \\
\angle G\left(j \omega_{c}\right)=\phi_{m}-\pi
\end{gathered}
$$

Sistemin yukarıda verilen özellikleri sağlaması için gerekli olan PI denetleyici bir sonraki bölümde tasarlanmıștır.

\section{TOPTD Sistemler için PI denetleyici tasarımı}

Bu bölümde TOPTD sistemler için PI denetleyicilerin analitik tasarım yöntemi ayrıntılı biçimde verilmiștir. Bir TOPTD model ve PI denetleyicinin frekans bölgesi gösterimi denklem (4) ve (5)'te verilmiști. Buradan yola çıkılarak üçüncü derece zaman gecikmeli transfer fonksiyonunun genlik ve fazı aşağıdaki gibi elde edilebilir.

$$
\begin{aligned}
P(j \omega) & =\left(\begin{array}{c}
-\frac{K\left(-1+T_{2} T_{3} \omega^{2}+T_{1}\left(T_{2}+T_{3}\right) \omega^{2}\right)}{\left(1+T_{1}^{2} \omega^{2}\right)\left(1+T_{2}^{2} \omega^{2}\right)\left(1+T_{3}^{2} \omega^{2}\right)} \\
+j\left(\frac{K \omega\left(-T_{2}-T_{3}+T_{1}\left(-1+T_{2} T_{3} \omega^{2}\right)\right)}{\left(1+T_{1}^{2} \omega^{2}\right)\left(1+T_{2}^{2} \omega^{2}\right)\left(1+T_{3}^{2} \omega^{2}\right)}\right)
\end{array}\right) \mathrm{e}^{-j L \omega} \\
& =|P(j \omega)| e^{j \angle P(j \omega)} \\
& =\sqrt{\frac{K^{2}}{\left(1+T_{1}^{2} \omega^{2}\right)\left(1+T_{2}^{2} \omega^{2}\right)\left(1+T_{3}^{2} \omega^{2}\right)}} \\
& \times e^{-j\left(\arctan \left(\frac{\omega\left(-T_{2}-T_{3}+T_{1}\left(-1+T_{2} T_{3} \omega^{2}\right)\right)}{-1+T_{2} T_{3} \omega^{2}+T_{1}\left(T_{2}+T_{3}\right) \omega^{2}}\right)+L \omega\right)}
\end{aligned}
$$

Buradan,

$$
\begin{gathered}
|P(j \omega)|=\sqrt{\frac{K^{2}}{\left(1+T_{1}^{2} \omega^{2}\right)\left(1+T_{2}^{2} \omega^{2}\right)\left(1+T_{3}^{2} \omega^{2}\right)}} \\
\angle P(j \omega)=-\arctan \left(\frac{\omega\left(-T_{2}-T_{3}+T_{1}\left(-1+T_{2} T_{3} \omega^{2}\right)\right)}{-1+T_{2} T_{3} \omega^{2}+T_{1}\left(T_{2}+T_{3}\right) \omega^{2}}\right)-L \omega
\end{gathered}
$$

olarak elde edilir. Benzer şekilde PI denetleyicinin frekans bölgesi genlik ve faz denklemleri aşağıdaki gibi elde edilir.

$$
\begin{aligned}
& |C(j \omega)|=\sqrt{k_{p}{ }^{2}+\left(-\frac{k_{i}}{\omega}\right)^{2}}=\sqrt{\frac{k_{i}^{2}+k_{p}{ }^{2} \omega^{2}}{\omega^{2}}} \\
& \angle C(j \omega)=\arctan \left(\frac{-\frac{k_{i}}{\omega}}{k_{p}}\right)=-\arctan \left(\frac{k_{i}}{k_{p} \omega}\right)
\end{aligned}
$$

Model ve denetleyicinin ayrı ayrı elde edilen frekans denklemleri ile sistemin genlik ve faz denklemleri aşağıda verildiği gibi yazılabilir.

$$
\begin{gathered}
|G(j \omega)|=|C(j \omega) P(j \omega)|=|C(j \omega)||P(j \omega)| \\
\angle G(j \omega)=\angle C(j \omega) P(j \omega)=\angle C(j \omega)+\angle P(j \omega)
\end{gathered}
$$

$\omega$ yerine $\omega_{c}$ yazılarak denklem (7)'de verilen sistem kazanç özelliği ele alındığında,

$$
\begin{aligned}
& \left|G\left(j \omega_{c}\right)\right|=\left|C\left(j \omega_{c}\right)\right|\left|P\left(j \omega_{c}\right)\right| \\
& =\sqrt{\frac{k_{i}^{2}+k_{p}^{2} \omega_{c}^{2}}{\omega_{c}^{2}}} \sqrt{\frac{K^{2}}{\left(1+T_{1}^{2} \omega_{c}^{2}\right)\left(1+T_{2}^{2} \omega_{c}^{2}\right)\left(1+T_{3}^{2} \omega_{c}^{2}\right)}}=1
\end{aligned}
$$

elde edilir. Benzer şekilde denklem (8)'deki sistem faz özelliği göz önüne alınarak,

$$
\begin{aligned}
& \angle G\left(j \omega_{c}\right)=\angle C\left(j \omega_{c}\right)+\angle P\left(j \omega_{c}\right) \\
& =-\arctan \left(\frac{k_{i}}{k_{p} \omega_{c}}\right) \\
& -\arctan \left(\frac{\omega_{c}\left(-T_{2}-T_{3}+T_{1}\left(-1+T_{2} T_{3} \omega_{c}^{2}\right)\right)}{-1+T_{2} T_{3} \omega_{c}^{2}+T_{1}\left(T_{2}+T_{3}\right) \omega_{c}^{2}}\right) \\
& -L \omega_{c}=\phi_{m}-\pi
\end{aligned}
$$

denklemi elde edilir. Denklem (19) ve (20)'nin beraber çözümünden PI denetleyici katsayıları aşağıda verildiği gibi elde edilir.

$$
\begin{gathered}
k_{p}= \pm \frac{\sqrt{1+T_{1}^{2} \omega_{c}^{2}} \sqrt{1+T_{2}^{2} \omega_{c}^{2}} \sqrt{1+T_{3}^{2} \omega_{c}^{2}}}{K \sqrt{1+\tan (\varphi)^{2}}} \\
k_{i}=\mp \frac{\omega_{c} \sqrt{1+T_{1}^{2} \omega_{c}^{2}} \sqrt{1+T_{2}^{2} \omega_{c}^{2}} \sqrt{1+T_{3}^{2} \omega_{c}^{2}} \tan (\varphi)}{K \sqrt{1+\tan (\varphi)^{2}}}
\end{gathered}
$$

Burada,

$$
\varphi=\phi_{m}-\pi+\arctan \left(\frac{\omega_{c}\left(-T_{2}-T_{3}+T_{1}\left(-1+T_{2} T_{3} \omega_{c}^{2}\right)\right)}{-1+T_{2} T_{3} \omega_{c}^{2}+T_{1}\left(T_{2}+T_{3}\right) \omega_{c}^{2}}\right)+L \omega_{c}
$$

olarak alınmalıdır. Bilindiği gibi zaman gecikmeli model analizi için literatürde farklı yöntemler bulunmaktadır. Bunlardan birisi de Smith Predictor yapısıdır. Bu yapıda, kapalı çevrim modelde bir değişiklik yapılarak sisteme bir tahminci eklenmektedir. Burada amaç, zaman gecikmesini çevrim dışına almaktır. Böylece işlem kolaylığı sağlanacaktır. Bu yayındaki yöntemde ise zaman gecikmesi olduğu gibi analize dahil edilmiş, trigonometrik eșdeğeri kullanılarak denklemler hesaplanmıștır. Bir sonraki bölümde, önerilen denklemler iki farklı zaman gecikmeli model üzerinde uygulanmıştır.

\section{Uygulama örnekleri}

$\mathrm{Bu}$ bölümde farklı TOPTD modeller üzerinden önerilen denetleyici tasarım yöntemi test edilmiştir.

Örnek 1: Așağıda verilen TOPTD sistem modelini ele alalım [6]. Bu örnek için istenen özellikler $\omega_{c}=0.1 \mathrm{rad} / \mathrm{sn}$ ve $\phi_{m}=45^{\circ}$ 
olarak seçilmiştir. Teoremde verilen denklemler kullanılarak aşağıdaki PI denetleyici elde edilmiştir.

$$
\begin{gathered}
P_{1}(s)=\frac{100}{(2 s+1)(5 s+1)(10 s+1)} \mathrm{e}^{-0.2 s} \\
C_{1}(s)=0.0102+\frac{0.0013}{s}
\end{gathered}
$$

$G_{1}(s)=C_{1}(s) P_{1}(s)$ sistemine ait Bode diyagramı Şekil 3'te verilmiştir.

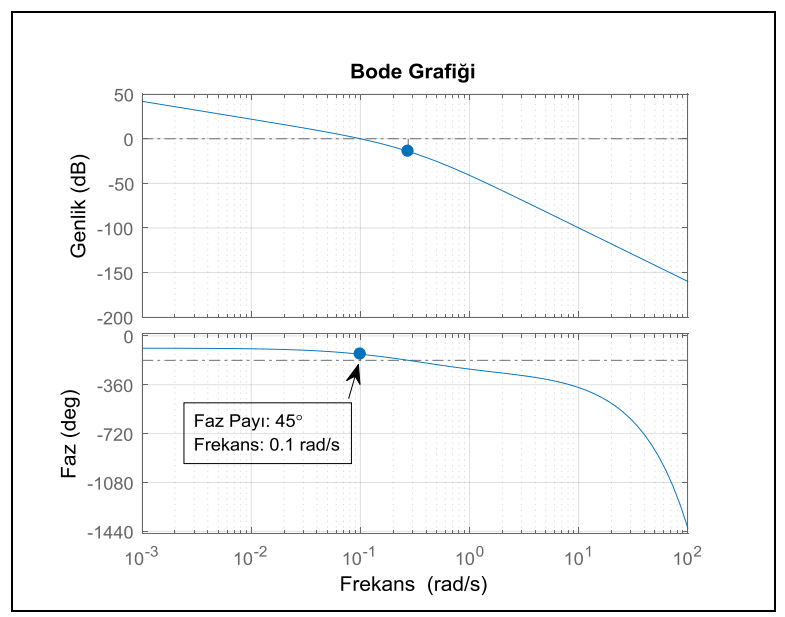

Şekil 3. $G_{1}(s)=C_{1}(s) P_{1}(s)$ sistemine ait Bode grafiği.

Figure 3. Bode plot of the system $G_{1}(s)=C_{1}(s) P_{1}(s)$.

Şekilden görüldüğü üzere istenen $\omega_{c}$ ve $\phi_{m}$ değerleri elde edilmiștir. Sistem kararlılığını incelemek için de birim basamak cevabı grafiği kullanılabilir. Şekil 4'te $G_{1}(s)=C_{1}(s) P_{1}(s)$ sistemine ait kapalı çevrim birim basamak cevabı gösterilmiștir.

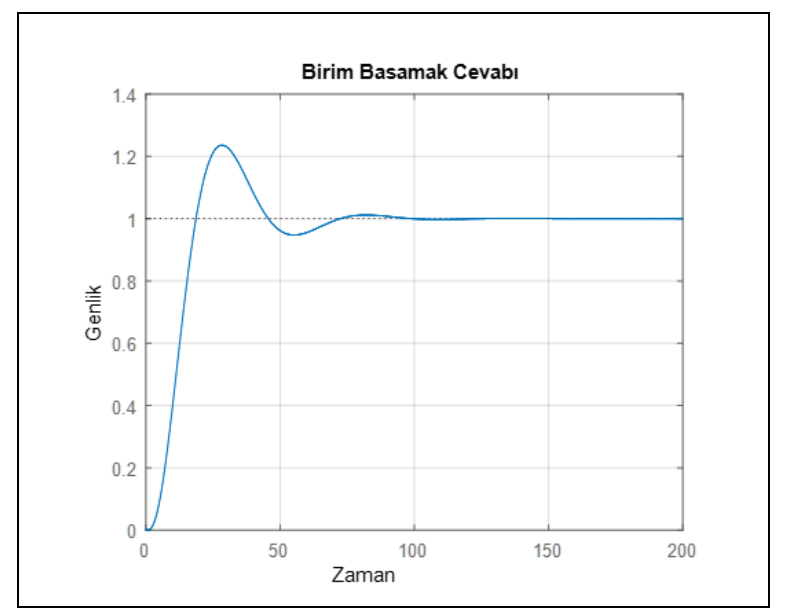

Şekil 4. $G_{1}(s)=C_{1}(s) P_{1}(s)$ sistemine ait birim basamak cevabı.

Figure 4. Step response of the system $G_{1}(s)=C_{1}(s) P_{1}(s)$.

Sistem dayanıklılığını test etmek amacıyla sistem kazancı $\pm \% 10$ oranında ileri ve geri değiștirilmiș ve elde edilen birim basamak cevabı da Şekil 5'te verilmiştir. Şekil 5'ten görüldüğü üzere sistem birim basamak cevabında $\pm \% 10$ kazanç değișiminde önemli değişiklikler görülmemiștir. Bu da kazanç değişimine karşı sistemin dayanıklılığını göstermektedir.

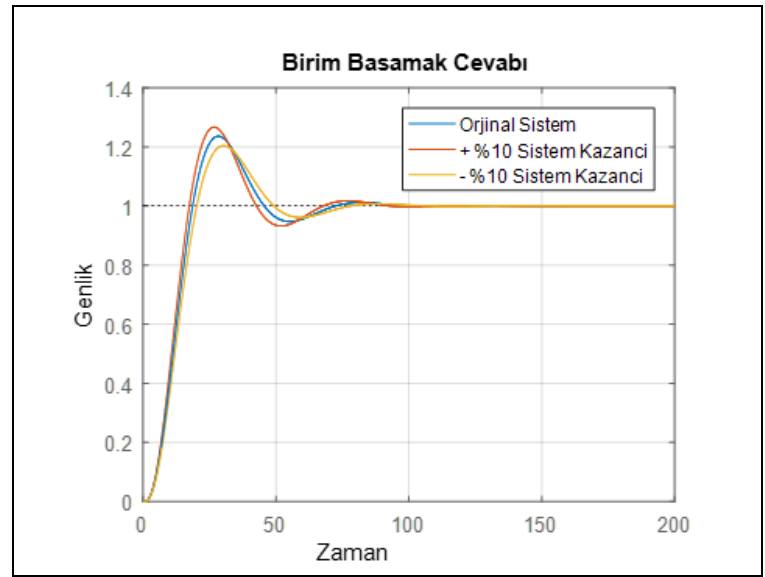

Şekil 5. Sistem kazancının $\pm \% 10$ değişimi ile elde edilen birim basamak tepkisi.

Figure 5. Step response obtained with $\pm \% 10$ iteration of the system gain.

Yukarıda faz payının $\phi_{m}=45^{\circ}$ olarak seçildiği durumda elde edilen Bode ve birim basamak cevapları verildi. Faz payının $\phi_{m} \in\left(10^{\circ} \rightarrow 90^{\circ}\right)$ aralığında $10^{\circ}$ artışıyla elde edilen denetleyici parametreleri Tablo 1'de gösterilmiştir.

Tablo 1'de verilen denetleyiciler ile elde edilen Bode grafikleri ise Şekil 6'da gösterilmiștir.

Tablo 1. Örnek 1 için $\phi_{m} \in\left(10^{\circ} \rightarrow 90^{\circ}\right)$ aralığında elde edilen denetleyici parametreleri.

Table 1. Controller parameters obtained in the interval $\phi_{m} \in\left(10^{\circ} \rightarrow 90^{\circ}\right)$ for example 1 .

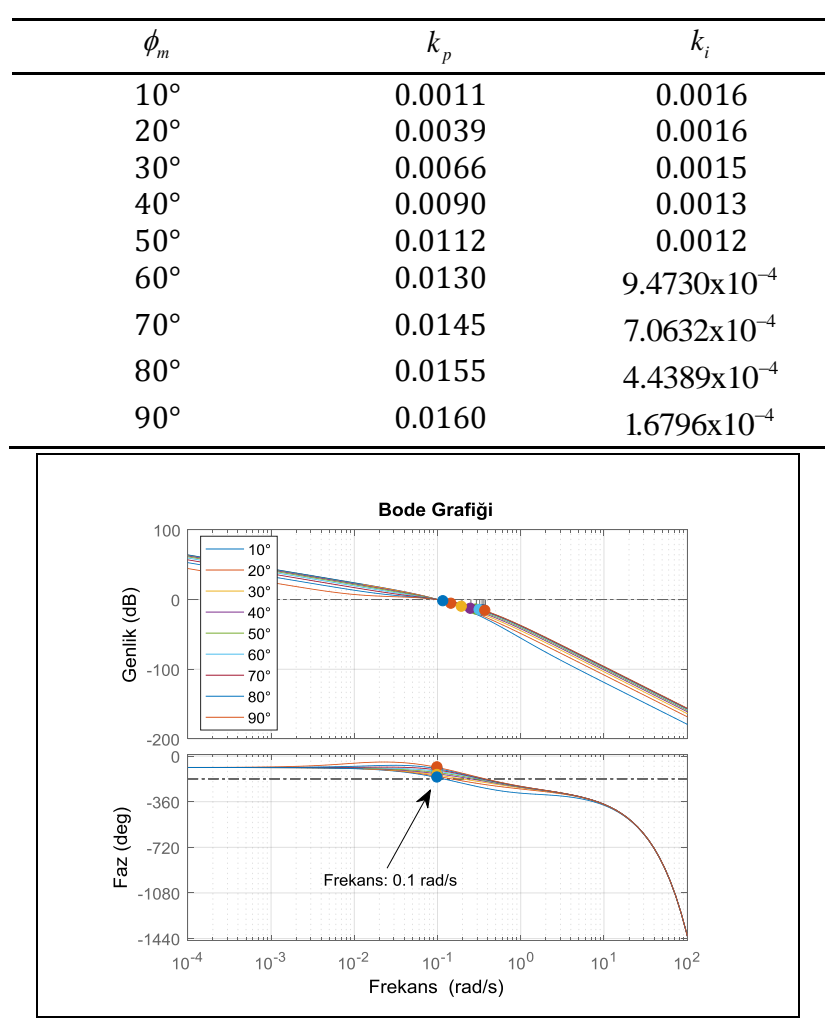

Şekil 6. Tablo 1'de verilen sistemlere ait Bode grafikleri.

Figure 6. Bode plots of the systems given in Table 1. 
Görüldüğü gibi Bode grafikleri verilen tüm sistemler, istenen kazanç kesim frekansı ve faz payı özelliklerini sağlamaktadır. Bilindiği gibi her sisteme özgü bir kararlılık frekans aralığ bulunmaktadır. Önerilen denklemlerle, daha büyük $\omega_{c}$ değerleri için farklı denetleyici parametreleri elde edilecektir fakat sistem kararlılığı garanti edilmemektedir. Sistem kararlılığı için uygun $\omega_{c}$ ve $\phi_{m}$ değerleri seçilmelidir. Bunun için SBL gibi farklı analiz yöntemleri bulunmaktadır. Önerilen denetleyici parametrelerini bir başka TOPTD model üzerinde test etmek faydalı olacaktır.

Örnek 2: Aşağıdaki TOPTD modeli ele alalım.

$$
P_{2}(s)=\frac{0.5}{(0.95 s+1)(1.1 s+1)(s+1)} \mathrm{e}^{-0.02 s}
$$

$\mathrm{Bu}$ örnek için istenen kazanç kesim frekansı $\omega_{c}=0.5 \mathrm{rad} / \mathrm{sn}$ olarak seçilmiştir. $\phi_{m} \in\left(10^{\circ} \rightarrow 90^{\circ}\right)$ aralığında elde edilen PI denetleyici parametreleri Tablo 2'de verilmiştir.

Tablo 2. Örnek 2 için $\phi_{m} \in\left(10^{\circ} \rightarrow 90^{\circ}\right)$ aralığında elde edilen denetleyici parametreleri.

Table 2. Controller parameters obtained in the interval $\phi_{m} \in\left(10^{\circ} \rightarrow 90^{\circ}\right)$ for example 2.

\begin{tabular}{ccc}
\hline$\phi_{m}$ & $k_{p}$ & $k_{i}$ \\
\hline $10^{\circ}$ & 0.0669 & 1.4122 \\
$20^{\circ}$ & 0.5563 & 1.3850 \\
$30^{\circ}$ & 1.0289 & 1.3156 \\
$40^{\circ}$ & 1.4701 & 1.2063 \\
$50^{\circ}$ & 1.8667 & 1.0603 \\
$60^{\circ}$ & 2.2066 & 0.8821 \\
$70^{\circ}$ & 2.4795 & 0.6771 \\
$80^{\circ}$ & 2.6770 & 0.4516 \\
$90^{\circ}$ & 2.7931 & 0.2123 \\
\hline
\end{tabular}

Tablodan faz payı $50^{\circ}$ olduğunda elde edilen denetleyici parametreleri kullanılarak $G_{2}(s)=C_{2}(s) P_{2}(s) \quad$ sistemi olușturulabilir. Söz konusu sisteme ait birim basamak cevabı ve $\pm \% 10$ kazanç değişimi ile elde edilen birim basamak cevapları Şekil 7'de verilmiştir.

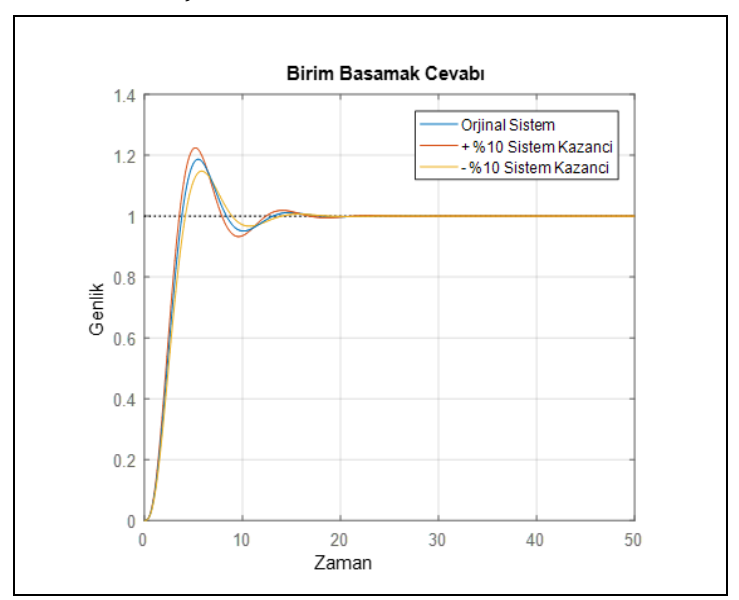

Şekil 7. Sistem kazancının $\pm \% 10$ değișimi ile elde edilen birim basamak tepkisi.

Figure 7. Step response obtained with $\pm \% 10$ iteration of the system gain.
Görüldüğü gibi, sistem dayanıklılığı başarılı bir şekilde sağlanmıștır. Tablo 2'de verilen denetleyiciler ile elde edilen sistemlerin Bode grafikleri ve birim basamak cevapları sırasıyla Şekil 8 ve Şekil 9'da gösterilmiştir.

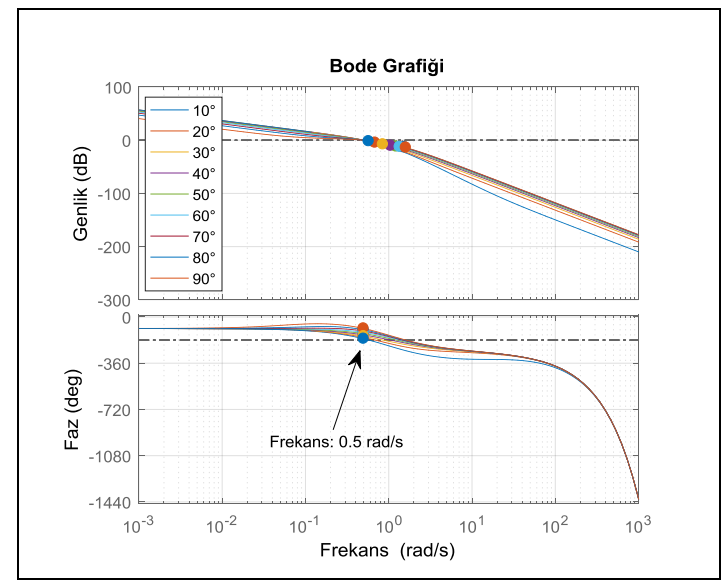

Şekil 8. Tablo 2'de verilen sistemlere ait Bode grafikleri.

Figure 8. Bode plots of the systems given in Table 2.

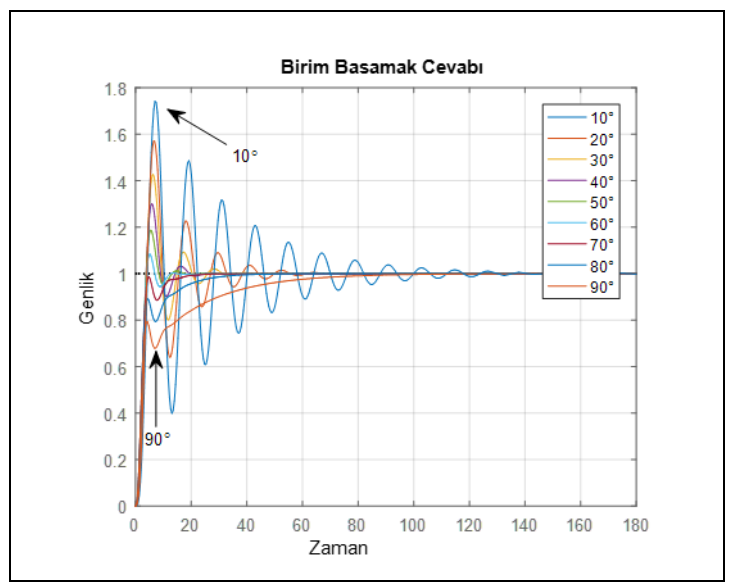

Şekil 9. Tablo 2'de verilen sistemlere ait birim basamak tepkileri.

Figure 9. Step responses of the systems given in Table 2.

Yukarıdaki şekillerden görüldüğü gibi tasarlanan denetleyiciler hem istenen kazanç ve faz özelliklerini hem de kararlılı̆̆ sağlamaktadır. Bu da TOPTD modeller için önerilen yöntemin etkinliğini ortaya koymaktadır.

\section{Sonuçlar}

Bu yayında zaman gecikmesi içeren üçüncü dereceden sistem modellerinin kararlılık, performans ve dayanıklılığı için oransal integral denetleyici tasarım yöntemi sunulmuştur. Önerilen yöntem, bu sistemler için genelleştirilmiş denetleyici parametre denklemlerini vermektedir. Denetleyici tasarımı, ideal bir sistemin frekans bölgesindeki kazanç kesim frekansı ve faz payı özelliklerinden yola çıkılarak yapılmıştır. Elde edilen sonuçlar grafiksel olarak ayrıntılı biçimde verilmiştir. Daha sonraki çalışmalarda kazanç kesim frekansı ve faz payı özelliklerinin yanısıra faz kesim frekansı ve kazanç payı özelliklerinin de sağlandığı denetleyici tasarımı ele alınabilir. 


\section{Conclusions}

In this paper, a proportional integral controller design method is presented for stability, performance and robustness of third order models including time delay. Proposed method gives the generalized parameter equations for such systems. Controller design is based on the gain crossover frequency and phase margin specifications of an ideal system in the frequency domain. Results obtained are illustratively given in detail. For future works, besides the gain crossover frequency and phase margin specifications, phase crossover frequency and gain margin specifications should be considered.

\section{Kaynaklar}

[1] Camcioğlu Ş, Özyurt B, Doğan İC, Hapoğlu H. "Application of response surface methodology as a new PID tuning method in an electrocoagulation process control case". Water Science \& Technology, 76(12), 3410-3427, 2017.

[2] Álvarez de Miguel S, Mollocana Lara JG, García Cena CE, Romero M, García de María JM, González-Aguilar J, "Identification model and PI and PID controller design for a novel electric air heater". Automatika, 58(1), 55-68, 2017.

[3] Sung SW, Je CH, Lee J, Lee, DH. "Improved system identification method for Hammerstein-Wiener processes". Korean Journal of Chemical Engineering, 25(4), 631-636, 2008.

[4] Rivas-Perez R, Castillo-Garcia F, Sotomayor-Moriano J, Feliu-Batlle V. "Design of a fractional order PI controller for steam pressure in the steam drum of a bagasse fired boiler". IFAC Proceedings, 47(3), 1337-1342, 2014.

[5] Malwatkar GM, Sonawane SH, Waghmare LM. "Tuning PID controllers for higher-order oscillatory systems with improved performance". ISA Transactions, 48(3), 347-353, 2009.

[6] León de la Barra BA, Jin L, Kim YC, Mossberg M. "Identification of first-order time-delay systems using two different pulse inputs". Proceedings of the 17th IFAC World Congress, Seoul, Korea, 6-11 July, 2008.

[7] Vivek S, Chidambaram M. "An improved relay auto tuning of PID controllers for critically damped SOPTD systems". Chemical Engineering Communications, 199(11), 1437-1462, 2012.

[8] Park HC, Lee J. "Step and pulse response methods for identification of wiener processes". American Institute of Chemical Engineers Journal, 52(2), 668-677, 2006.

[9] Cheon YJ, Jeon CH, Lee, J, Sung SW. "Improved fourier transform to estimate frequency responses". Korean Journal of Chemical Engineering, 26(4), 925-929, 2009.

[10] Darwish NM. "PID controller design in the frequency domain for time-delay systems using direct method". Transactions of the Institute of Measurement and Control, 40(3), 940-950, 2018.

[11] Alabi SB, Ikpatt FE. "Computer-aided simplification of high-order linear models". Indian Chemical Engineer, 51(2), 129-155, 2009.
[12] Sam YM, Osman JHS, Ruddin M, Ghani A. "A class of proportional-integral sliding mode control with application to active suspension system". Systems \& Control Letters, 51(3-4), 217-223, 2004.

[13] Bhatti SA, Malik SA, Daraz A. "Comparison of P-I and I-P controller by using Ziegler-Nichols tuning method for speed control of DC motor". 2016 International Conference on Intelligent Systems Engineering, Islamabad, India, 15-17 January, 2016.

[14] Sönmez S, Ayasun S. "Stability region in the parameter space of PI controller for a single-area load frequency control system with time delay". IEEE Transactions on Power Systems, 31(1), 829-830, 2016.

[15] Khooban MH, Niknam T, Sha-Sadeghi M. "Speed control of electrical vehicles: a time-varying proportional-integral controller-based type-2 fuzzy logic". IET Science, Measurement \& Technology, 10(3), 185-192, 2016.

[16] Vishnu MJ, Karthika S. "Comparison of speed control of PMSM using adaptive fuzzy logic controller and PI controller". Journal of Power Electronics \& Power Systems, 8(3), 24-30, 2018.

[17] Karthika S, Vishnu MJ. “Comparative study of torque ripple minimization of PMSM using PI and proportional resonant (PI-RES) controller". Journal of Power Electronics \& Power Systems, 8(3), 17-23, 2018.

[18] Shafie Z, Shenton AT. "Frequency domain design of PID controllers for stable and unstable systems with time delay". Automatica, 33(12), 2223-2232, 1997.

[19] Tan N, Kaya I, Celaleddin Y, Atherton DP. "Computation of stabilizing PI and PID controllers using the stability boundary locus". Energy Conversion and Management, 47, 3045-3058, 2006.

[20] Hamamci SE, Tan N. "Design of PI controllers for achieving time and frequency domain specifications simultaneously". ISA Transactions, 45(4), 529-543, 2006.

[21] Şenol B, Demiroğlu U. "Frequency frame approach on loop shaping of first order plus time delay systems using fractional order PI controller". ISA Transactions, 86, 192-200, 2019.

[22] Şenol B, Demiroğlu U. "Fractional order proportional derivative control for first order plus time delay plants: achieving phase and gain specifications simultaneously". Transactions of the Institute of Measurement and Control, 41(15), 4358-4369, 2019.

[23] Wang C, Jin Y, Chen YQ. "Auto-tuning of FOPI and FO[PI] controllers with iso-damping property". Proceedings of the 48h IEEE Conference on Decision and Control (CDC) held jointly with $200928^{\text {th }}$ Chinese Control Conference, Shanghai, China, 15-18 December 2009.

[24] Wang C, Luo Y, Chen YQ. "Fractional order proportional integral (FOPI) and [proportional integral] (FO[PI]) controller designs for first order plus time delay (FOPTD) systems". Chinese Control and Decision Conference, Guilin, China, 17-19 June 2009. 